\title{
Growth Profiles of Human Autosomal Trisomies at Midgestation
}

\author{
MASON BARR, JR. \\ Departments of Pediatrics, Pathology, and Obstetrics and Gynecology, University of Michigan, \\ Ann Arbor, Michigan 48109
}

\begin{abstract}
Somatic and visceral growth profiles of midgestation human fetuses with trisomy 21,18 , or 13 demonstrate that each disorder has a characteristic pattern of growth aberration. The most striking deviations are short limbs in trisomy 21 , subnormal adrenal and lung weights in trisomy 18 , and supranormal spleen and kidney weights in trisomy 13. (c) 1994 Wiley-liss, Ine
\end{abstract}

"Dysmorphologists . . could pay attention to the dysmorphology of the unborn and his membranes in order to recognize the circumstances which lead to elimination of abnormal embryos and maintenance of the normal" (Warkany, '78).

In the consideration of the pathology of the common human autosomal trisomies (trisomies 21, 18, and 13), structural abnormalities have received the lion's share of attention. All three are known to be associated with varying degrees of intrauterine growth restriction, and trisomy 18 has often been cited as an example of "symmetrical" growth restriction. Most studies of growth in particular syndromes are concerned with its characterization in infants and children. Such studies typically include only height, weight, and head circumference. With the increasing use of high-resolution prenatal ultrasonography, the characterization of growth during gestation has assumed considerable importance. The definition not only of gross somatic measurements (e.g., biparietal diameter, femur length, head and abdominal circumferences) but also those of particular parts or organs (e.g., kidneys, cerebellum) has been recognized as relevant to the diagnosis of various syndromes and other fetal conditions.

Limb shortness in trisomy 21 has been recognized (Cuckle et al., '89; Fitzsimmons et al., '89; Rodis et al., '91; Benacerraf et al., '91; Rotmensch et al., '92; Nyberg et al., '93). In many centers it constitutes one of the prenatal screening clues for the suspicion of trisomy 21 . The birth weights and postnatal growth of trisomy 21 have been extensively studied (Pueschel et al., '76; Cronk, '78; Cronk et al., '88), but there is little systematic evaluation of growth profiles in midgestation at the time when ultrasonography for prenatal diagnosis is done. Aside from studies of birth weight and length of infants with several specified malformations ( $\mathrm{Ku}$ cera and Dolezalova, '72 and '73), there is little information on visceral growth in abnormal fetuses, particularly the common autosomal trisomies.

\section{MATERIALS AND METHODS}

Fetuses studied were from the perinatal pathology data set $(n=3053)$ of the University of Michigan Teratology Unit. Selection criteria were: absence of maceration, body weight between 100 and 1000 g, karyotype confirmed from amniocytes and / or fetal tissues, and morphology consistent with the cytogenetic diagnosis. From a pool of 89 cases of trisomy 21, 68 met the selection criteria; from a pool of 45 trisomy $18 \mathrm{~s}, 25$ met the criteria; and from a pool of 28 trisomy $13 \mathrm{~s}, 12$ met the criteria. Of these 12 , four had no holoprosencephaly and eight had alobar holoprosencephaly; the latter were compared with three cases with alobar holoprosencephaly that had normal karyotypes and no other malformations.

In order to determine the normality/abnormality of the morphometrics in aneuploid fetuses it was first necessary to develop standards for "normal" (more correctly, "typical") fetuses. This has been done and reported previously (Barr et al., '94). Using these growth parameters, Z-scores (observed minus expected, divided by the standard deviation) were computed for selected somatic measurements (body weight, crownheel and crown-rump lengths, head circumference, and arm, leg and foot lengths) and selected viscera weights (brain, spleen, thymus, thyroid, heart, liver, adrenals, kidneys, and lungs), using body weight and brain weight as the independent variables. Mean standard scores and their standard deviations for each syndrome were computed. Although statistical testing for significant differences from the expected values was done, using two-tailed t-tests, this seemed to overstate the case for growth aberration simply because of the large

Received September 12, 1994; accepted November 21, 1994.

Address reprint requests to Mason Barr, Jr., M.D., Pediatric-Genetics-Teratology Unit, D1109 MPB 0718, University of Michigan Medical Center, Ann Arbor, MI 48109-0718. 
sample size of the controls (n 1014). Accordingly, probability values are not displayed in the figures, but the reader is left to visually assess the magnitude of the deviations of the observed means from those expected. It is felt that a suitable criterion for biologically meaningful aberration is an observed mean greater than one standard deviation above or below the expected mean.

\section{RESULTS}

In trisomy 21 (Fig. 1), there is an overall depression of both somatic measurements and visceral weights by body weight standards. This is judged to be the result of mild edema often present at this stage of gestation (although the edema may, on occasion, be severe enough to qualify as hydrops fetalis). When judged by brain weight standards, somatic measurements tend to be elevated, with the exception that limb lengths are reduced. As noted by others, the limb length reduction in trisomy 21 is more pronounced for the arms than the legs. It is also noted that there is a discordance between head circumference and brain weight; this is correlated with an apparent excess of extracerebral CSF, although fluid volumes have not actually been measured to verify this observation. When visceral weights are considered by brain weight standards, the major deviations are depressions of adrenal and lung weights. These reductions, while statistically significant, are not of a degree that would predict any particular clinical correlates for the neonate with trisomy 21, nor, except for the short limbs, would they be detectable by prenatal ultrasonography.

In trisomy 18 (Fig. 2), deviations from normal morphometrics are more pronounced than in trisomy 21 . Both trunk and limbs are appreciably shorter. Head circumference is mildly elevated for body weight, and this is accompanied by a brain weight that is also excessive for body weight. Among the fetuses examined there were no instances of hydrocephaly. The phenotype of trisomy 18 is not uncommonly described as including a small face. These data suggest the problem may be more accurately the disproportion between head and face, with the head size matching gestational age and the face matching the small body. Among the viscera in trisomy 18, spleen weight is elevated (although the variance is rather large), while liver, adrenal and lung weights are reduced.

The analysis of trisomy 13 fetuses (Fig. 3) was confounded by the presence or absence of alobar holoprosencephaly. Accordingly, the sample of fetuses with trisomy 13 was subdivided based on this brain malformation. Those without holoprosencephaly have rather normal somatic measurements, although their head circumferences and brain weights are demonstrably subnormal. In this subset, visceral weight aberrations are most pronounced for splenomegaly and renomegaly (note that no cases of cystic renal dysplasia were included in the sample). Among trisomy 13 fetuses with

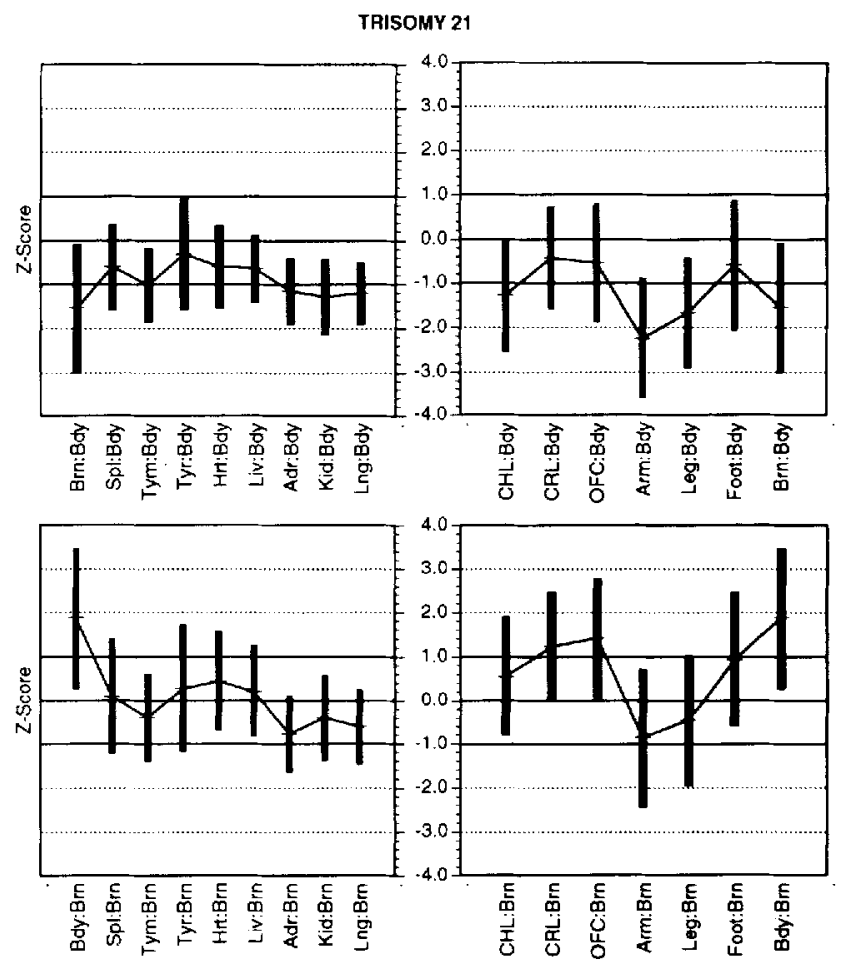

Fig. 1. Growth profiles for trisomy 21.

Bdy body weight

CHL crown-heel length

CRL crown-rump length

OFC head circumference

Arm arm length

Leg leg length

FTL foot length

Brn brain weight

Spl spleen weight

Tym thymus weight

Tyr thyroid weight

Hrt heart weight

Liv liver weight

Adr adrenal weight (combined)

Kid kidney weight (combined)

Lng lung weight (combined).

Details of measurements are as in Barr et al. ('94). On the X-axis, the independent variable is placed before the colon and the dependent variable (or standard) after.

The bars represent the observed mean \pm one standard deviation; the comparable range for the control fetuses is the distance between a $\mathrm{Z}$-score of 1.0 to -1.0 , with $\mathrm{Z}=0$ being the expected mean. The bars are connected only to emphasize the pattern when comparing one syndrome with another (Poznanski et al., '72).

alobar holoprosencephaly, head circumference and brain weight are drastically reduced, as expected.* Loss of normal cranial dimensions is also reflected in a decreased crown-rump length. The splenomegaly and renomegally seen in the nonholoprosencephalic subset are found in these fetuses as well (again no cases of

* However, with alobar holoprosencephaly head circumference may be quite variable, spanning the range from macrocephalic (hydrocephaly) to profound microcephaly. 


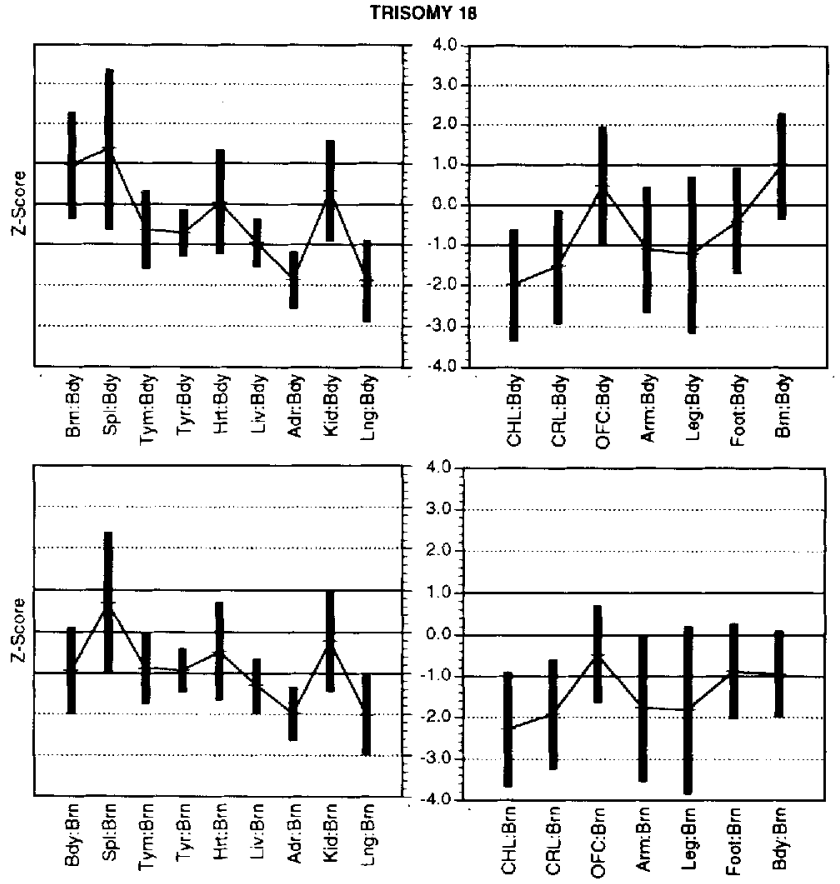

Fig. 2. Growth profiles for trisomy 18. See legend of Fig. 1 for explanation.

cystic renal dysplasia were included in the sample). In both subsets, thymus and heart weights are reduced, while lung weight is reduced only in those with the major brain anomaly.

\section{DISCUSSION}

"Phenotype" typically refers to structural features, although for some syndromes growth and functional features are key components. Many factors influence prenatal growth, including heritable factors, environmental factors, and factors intrinsic to the conceptus. Growth aberrations may be somatic or visceral, generalized or localized, transient or permanent, accelerated or restricted, and/or of early or late onset. They may be correlated, singly or in combination, with structural abnormalities, functional abnormalities, death, or premature delivery.

What is to be made of the growth data presented here? Some of the aberrations are of such a nature that they could be useful in the in utero ultrasonographic screening of fetuses for autosomal trisomy. This is already the case for trisomy 21 , the most prevalent of the trisomies at midgestation. The shortness of both the arms and legs has been noted, but by themselves short limbs are not highly specific indicators of trisomy 21 (Cuckle et al., '89: Fitzsimmons et al., '89; Rodis et al., '91; Benacerraf et al., '91; Rotmensch et al., '92; Nyberg et al., '93). To reduce the number of women advised to undergo amniocentesis, while maintaining or increasing the detection of trisomy 21 , various combinations of
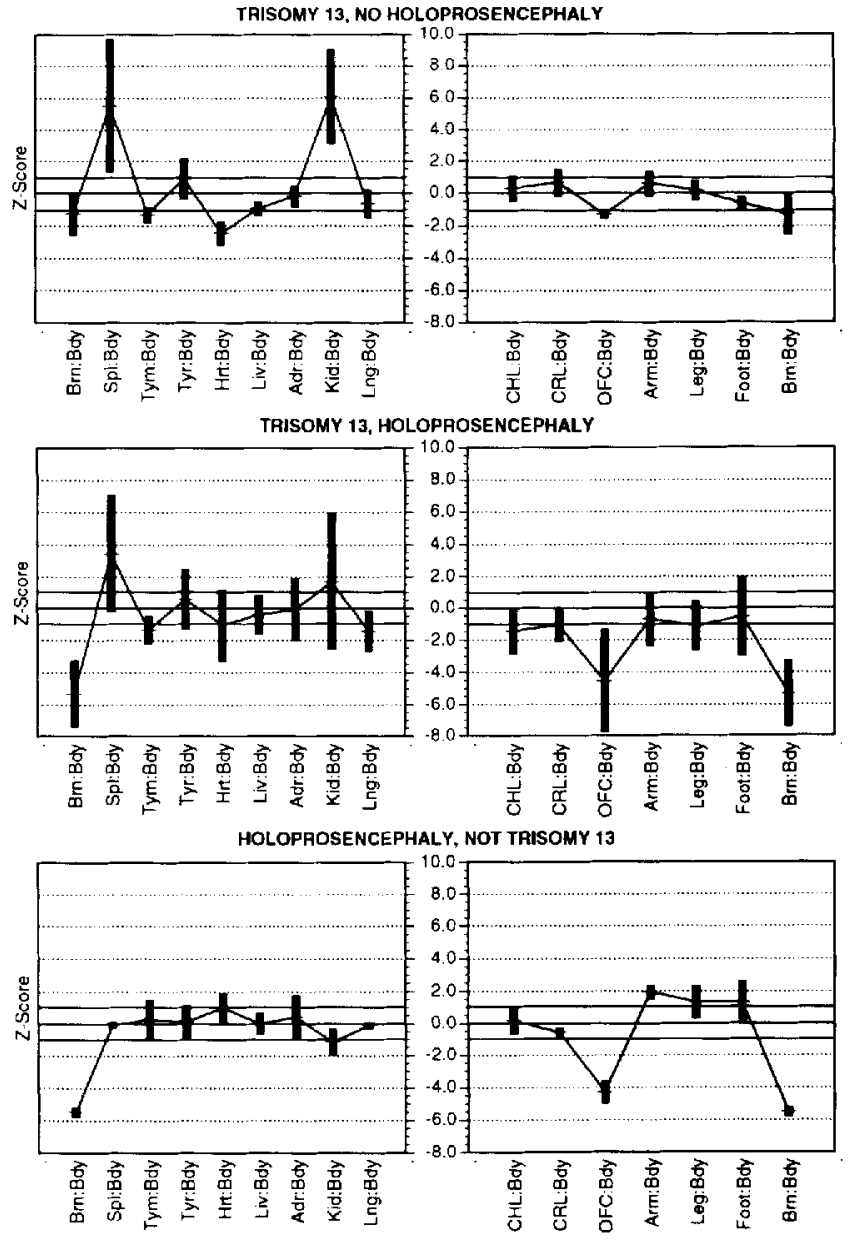

Fig. 3. Growth profiles for trisomy 13, with and without alobar holoprosencephaly, and for euploid fetuses with alobar holoprosencephaly. See legend of Fig. 1 for explanation.

limb measurements have been proposed to increase the specificity and sensitivity of ultrasonographic screening (Johnson et al., '93a, '93b, 94).

While growth aberrations may most commonly be inherent to the genotype, it is suspected that at least some could be reflections of altered fetal physiology. Such disturbances in fetal physiology may not be specific to any one syndrome, but might have general application irrespective of the syndrome. If that is true, finding a particular growth anomaly might be a tipoff to a physiologic derangement and allow a focused approach to fetal therapy.

Some of the observed patterns suggest the need for investigation of the interaction between physiology and growth of the trisomic fetus. In the case of trisomy 13 fetuses, could reduced lung weight be a consequence of reduced fetal breathing activity associated with holoprosencephaly? On the other hand, fetuses with isolated alobar holoprosencephaly do not show evidence of lung undergrowth. Other than having comparable degrees of reduced head circumference and brain weight, 
euploid fetuses with holoprosencephaly show no growth pattern similarities to the trisomy 13 fetuses. The reason for the spenomegaly and renomegaly in trisomy 13 remains to be discovered.

Trisomy 18 is often cited as an archetypal example of "symmetrical" intrauterine growth restriction. These data indicate that in trisomy 18 , at midgestation, the growth aberration is anything but symmetrical (Johnson et al., '89). The lung and adrenal weights are often reduced to a degree that suggests the neonate with trisomy 18 may experience physiological problems with lung and adrenal function. This may in turn contribute to the early neonatal lethality of this syndrome.

It is to be noted that each of the three autosomal trisomies covered in this report has its own distinctive pattern of somatic and visceral growth. These patterns have been helpful in making a diagnosis in fetuses submitted for examination when no material for cytogenetic study is available (i.e., formalin fixation or severe maceration). It is cautioned that the patterns reported here apply only to the midgestation fetus. There is some evidence that the characteristics of these patterns may change by term, reflecting some potential for either "catch-up" growth or later-onset growth restriction (Johnson et al., '89). To date, the sample size of aneuploid term infants from my collection is insufficient to make any definitive assessment of these possibilities.

The finding of an autosomal trisomy is often considered an adequate explanation of fetal or neonatal death. However, as pointed out by Rushton ('85), the mere existence of a karyotypic anomaly does not really explain why that particular death occurred. We should be better informed if we understood whether or not the presence of small adrenals, small hearts, small lungs, large spleens, or large kidneys has anything to do with the clinical course. If, as Hook ('79) has estimated, $23 \%$ of trisomy 21 fetuses die between midgestation and term, our question should be why and how do they die? Warkany (78) coined the term terathanasia in recognition of the general lethality of many malformations and anomalies. He suggested that, if nature generally aborts abnormal fetuses, perhaps the survival of some represents a failure of the natural screening process and the reasons for this failure would be worthy of study. The data from this study do not permit a distinction between spontaneously and medically aborted fetuses, but they do extend the description of the dysmorphology of the autosomal trisomies. The finding of aberrant growth patterns suggests additional useful points to look for answers to Warkany's challenge.

\section{LITERATURE CITED}

Barr, M., W.R. Blackburn, and N.R. Cooley (1994) Human fetal somatic and visceral morphometrics. Teratology, 49:487-496.
Benacerraf, B.R., D. Neuberg, and F.D. Frigoletto (1991) Humeral shortening in second trimester fetuses with Down syndrome. Obstet. Gynecol., 77:223-227.

Cronk, C.E. (1978) Growth of children with Down's syndrome: Birth to age 3 years. Pediatrics, $61: 564-568$.

Cronk, C., A.C. Crocker, S.M. Pueschel, A.M. Shea, E. Zackai, G. Pickens, and R.B. Reed (1988) Growth charts for children with Down syndrome: 1 month to 18 years of age. Pediatrics, 81:102110.

Cuckle, H., N. Wald, J. Quinn, P. Royston, and L. Butler (1989) U1trasound fetal femur length measurement in the screening for Down's syndrome. Br. J. Obstet. Gynaecol., 96:1373-1378.

Fitzsimmons, J., S. Droste, T.H. Shepard, J. Pascoe-Mason, A. Chinn, and L.A. Mack (1989) Long-bone growth in fetuses with Down syndrome. Am. J. Obstet. Gynecol., 161:1174-1177.

Hook, E.B. (1979) Genetic counseling and prenatal cytogenetic services: Policy implications and detailed cost-benefit analyses of programs for the prevention of Down syndrome. In: Service and Education in Medical Genetics. I.H. Porter and E.B. Hook, eds. Academic Press, San Diego, pp. 29-54.

Johnson, M.P., M. Barr, F. Qureshi, A. Drugan, and M.I. Evans (1989) Symmetrical intrauterine growth retardation is not symmetrical: Organ-specific gravimetric deficits in midtrimester and neonatal trisomy 18. Fetal Ther., 4:110-119.

Johnson, M.P., M. Barr, M.C. Treadwell, J. Michaelson, N.B. Isada, P.G. Pryde, M.P. Dombrowski, D.B. Cotton, and M.I. Evans (1993a) Fetal leg and femur: foot length ratio: A marker for trisomy 21. Am. J. Obstet. Gynecol., 169:557-563.

Johnson, M.P., J.E. Michaelson, R. Ayoub, M. Barr, M.C. Treadwell, N.B. Isada, A. Reichler, R.F. Hume, and M.I. Evans (1993b) Brachycephaly alters biparietal diameter (BPD) in trisomy 21 (T21): Fetal foot length is a better gestational age measurement in sonographic morphologic screening. Am. J. Hum. Genet, 53:A1423.

Johnson, M.P., J.E. Michaelson, M. Barr, M.C. Treadwell, N.B. Isada, M.P. Dombrowski, P.G. Pryde, J. Addis, and M.I. Evans (1994) Sonographic screening for trisomy 21: Fetal humerus: foot length ratio, a useful new marker. Fetal Diagn. Ther., 9:130-138.

Kucera, J., and V. Dolezalova (1972) Prenatal development of malformed fetuses at 28-42 weeks of gestational age (anencephalus, hydrocephalus, Down's syndrome, cleft lip and palate, and hypospadias). I. Weight gains. Biol. Neonate, 20:253-261.

Kucera, J., and V. Dolezalova (1973) Prenatal development of malformed fetuses at 28-42 weeks of gestational age (anencephalus, hydrocephalus, Down's syndrome, cleft lip and palate, and hypospadias). II. Length gains. Biol. Neonate, 30:319-324.

Nyberg, D.A., R.G. Resta, D.A. Luthy, D.E. Hickok, and M.A. Williams (1993) Humerus and femur length shortening in the detection of Down's syndrome. Am. J. Obstet. Gynecol., 168:534-538.

Poznanski, A.K., S.M. Garn, J.M. Nagy, and J.C. Gall (1972) Metacarpophalangeal pattern profiles in the evaluation of skeletal malformation. Radiology, 104:1-11.

Pueschel, S.M., K.J. Rothman, and J.D. Ogilby (1976) Birthweight of children with Down's syndrome. Am. J. Ment. Defic., 80:442-445.

Rodis, J.F., A.M. Vintizileos, A.D. Fleming, L. Ciarleglio, A. Nardi, L. Feeney, W.E. Scorza, W.A. Campbell, and C. Ingardia (1991) Am. J. Obstet. Gynecol., 165:1051-1056.

Rotmensch, S., J.S. Luo, M. Liberati, K. Belanger, M.J. Mahoney, and J.C. Hobbins (1992) Fetal humeral length to detect Down syndrome. Am. J. Obstet. Gynecol., 166:1330-1334.

Rushton, D.I. (1985) The nature and causes of spontaneous abortions with normal karyotypes. In: Issues and Reviews in Teratology. Vol. 3, H. Kalter, ed. Plenum Press, New York, pp. 21-63.

Smith, A., and T. McKeown (1955) Pre-natal growth of mongoloid defectives. Arch. Dis. Child., 30:257-259.

Warkany, J. (1978) Terathanasia. Teratology, 17:187-192. 